\title{
Epidemiology of Pancreatic Cancer in Saudi
} Arabia: A Retrospective Analysis of Pancreatic \section{Cancer Diagnosed in Saudi Arabia Between 2004 and 2015}

This article was published in the following Dove Press journal:

Clinical and Experimental Gastroenterology

\author{
Ibrahim G Alghamdi' \\ Majid Almadi ${ }^{2,3}$ \\ Fahad Alsohaibani ${ }^{4}$ \\ Mahmoud Mosli ${ }^{5}$ \\ Edward B De Vol iD 6 \\ Faisal Abaalkhail ${ }^{7,8}$ \\ Faisal A AISaif ${ }^{9}$ \\ Waleed K Al-hamoudi ${ }^{10}$ \\ Nasser Al-Sanea ${ }^{11-13}$ \\ Mazen Hassanain 9,14 \\ Saleh A Alqahtani ${ }^{6,15,16}$
}

'Public Health Department, College of Applied Medical Sciences, University of Al-Baha, Al-Baha, Saudi Arabia; ${ }^{2}$ Division of Gastroenterology,

Department of Medicine, College of Medicine, King Khalid University Hospital, King Saud University,

Riyadh, Saudi Arabia; 'Division of Gastroenterology, McGill University Health Center, Montreal General

Hospital, McGill University, Montreal, Canada;

${ }^{4}$ Department of Medicine, King Faisal Specialist

Hospital \& Research Center, Riyadh, Saudi Arabia;

${ }^{5}$ Division of Gastroenterology, Department of

Medicine, King Abdulaziz University, Jeddah, Saudi

Arabia; ${ }^{6}$ Biostatistics, Epidemiology, and Scientific

Computing Department, King Faisal Specialist

Hospital \& Research Center, Riyadh, Saudi Arabia;

${ }^{7}$ Gastroenterology Section, Department of

Medicine, King Faisal Specialist Hospital and

Research Center, Riyadh, Saudi Arabia; ${ }^{8}$ College of

Medicine, Alfaisal University, Riyadh, Saudi Arabia;

${ }^{9}$ Department of Surgery, College of Medicine, King

Saud University, Riyadh, Saudi Arabia; ${ }^{10}$ Liver

Disease Research Center, College of Medicine, King

Saud University, Riyadh, Saudi Arabia; "Health

Affairs, Princess Nourah Bint Abdulrahman

University, Riyadh, Saudi Arabia; ${ }^{12}$ Department of

Surgery, College of Medicine, Alfaisal University

Riyadh, Saudi Arabia; ${ }^{13}$ Department of Surgery, King

Faisal Specialist Hospital \& Research Center, Riyadh,

Saudi Arabia; ${ }^{14}$ Department of Pediatrics, Baylor

College of Medicine, Houston, TX, USA; ${ }^{15}$ Liver

Transplant Center, King Faisal Specialist Hospital \&

Research Center, Riyadh, Saudi Arabia; ${ }^{16}$ Division of

Gastroenterology and Hepatology, Johns Hopkins

University, Baltimore, MD, USA
Correspondence: Saleh A Alqahtani Liver Transplant Center, King Faisal Specialist Hospital and Research Centre, Riyadh, Saudi Arabia

Email salalqahtani@kfshrc.edu.sa
Purpose: Over the last decades, the incidence of pancreatic cancer has increased, particularly in countries with a higher socioeconomic status. The present work aimed to provide detailed epidemiological data on the incidence of pancreatic cancer in Saudi Arabia.

Patients and Methods: In this retrospective descriptive study, the epidemiological data on pancreatic cancer cases diagnosed in 13 administrative regions of Saudi Arabia between January 2004 and December 2015 were extracted from the Saudi Cancer Registry. The frequency, the crude incidence rate (CIR), and the age-standardized incidence rate (ASIR), stratified by geographical region, gender, and the year of diagnosis, were analyzed.

Results: From January 2004 to December 2015, a total of 2338 cases of pancreatic cancer were registered, including 1443 males and 895 females. The overall CIR was 1.28/100,000 among males and 0.80/100,000 in females, with an overall ASIR of 2.26 and 1.41/100,000 for males and females, respectively. Higher ASIR and CIR were observed among males than females (ratio 1.6). In both genders, the ASIR of pancreatic cancer increased with increasing age, with the highest incidence in patients aged 70 years or more. The ASIR in the Eastern Region (3.2/100,000) and the regions of Riyadh (3.0/100,000) and Tabuk $(2.6 / 100,000)$ proved to be significantly higher than in the other regions of the country. Among women, the ASIR was significantly higher in Riyadh $(2.3 / 100,000)$, the northern region (2.2/ 100,000), and Tabuk (2.0/100,000).

Conclusion: This study revealed a slight increase of the CIR and ASIR of pancreatic cancer among males and females of the Saudi population. Eastern region, Riyadh, and Tabuk had the highest overall ASIRs of pancreatic cancer among males, Riyadh, Northern region, and Tabuk among Saudi females. The area least affected by pancreatic cancer was observed in Jazan among male and female Saudis. The rates of pancreatic cancer in Saudi Arabia were significantly higher among males compared with female Saudis. Further analytical studies are needed to identify the potential risk factors for pancreatic cancer among the Saudi population.

Keywords: pancreatic cancer, epidemiology, Saudi Arabia, incidence, Middle-East

\section{Introduction}

Pancreatic cancer is a notoriously aggressive tumor type that is associated with a very poor prognosis. In fact, the 5-year overall survival rate for patients with pancreatic cancer is very dismal at only about $6 \% .{ }^{1}$ The main reason for this poor 
prognosis lies in the fact that pancreatic cancer is usually diagnosed in an advanced stage, at which a curative treatment approach is no longer feasible. This is amply illustrated by the fact that only about $20 \%$ of newly diagnosed pancreatic cancer patients are able to undergo surgical resection with curative intent. ${ }^{1,2}$ For these patients, the survival prospects are somewhat better, with up to a quarter of patients surviving beyond 5 years. ${ }^{2}$ For patients with unresectable pancreatic cancer, therapeutic progress has been slow, with only marginal improvements in 5-year overall survival rates over the last decades.

In 2018, pancreatic cancer ranked 14th in the list of most frequently diagnosed cancers in the world but proved to be the 7th most common cause of cancerrelated death. ${ }^{3}$ Globally, the age-standardized incidence rate (ASIR) for pancreatic cancer was reported at 4.8/ 100,000 in 2018. Pancreatic cancer comes with a slight male predominance, with a global ASIR of 5.5/100,000 and 4.8/100,000 for men and women, respectively. ${ }^{4}$ In addition, pancreatic cancer is primarily a disease of the elderly. The incidence and mortality of pancreatic cancer increase with age, with the vast majority $(+90 \%)$ of patients being diagnosed above the age of 55 and median age at diagnosis of approximately 70 years. ${ }^{1,5}$ Over the last decades, the incidence of pancreatic cancer has steadily increased. In this respect, results from a global burden of disease study focusing on pancreatic cancer revealed that the global incidence of pancreatic cancer increased with a factor of 2.3 from 1990 to $2017 .^{5}$ Interestingly, across the different regions included in this analysis, the increased incidence of pancreatic cancer was found to be correlated with an increasing socioeconomic status. $^{5}$

A possible explanation for the higher incidence of pancreatic cancer in countries with a higher income and a better socioeconomic status could lie in the fact that people can attain an older age, which increases their risk of developing pancreatic cancer. Moreover, better access to medical care and more systematic cancer registration in these countries may also contribute to a higher reported incidence. In addition to this, evolutions in lifestyle choices, leading to higher exposure to risk factors, may also play their part. ${ }^{6,7,8}$

The particularly high mortality of pancreatic cancer represents an important challenge to the medical community and underscores the need for a better insight into the pathogenesis of this disease. Until now, there has not been a detailed epidemiological analysis of pancreatic cancer in
Saudi Arabia. According to the 2018 GLOBOCAN data, the burden of pancreatic cancer in the country is relatively low, with an incidence of 2.2/100,000 (ASIR: 2.7/100,000 in males and 1.6/100,000 for females) and the annual standardized rate for mortality of $1.6 / 100,000 .^{3}$ The aim of this study was to provide a detailed overview of the epidemiology of pancreatic cancer in Saudi Arabia. We evaluated the incidence of pancreatic cancer in men and women, looked into potential regional differences, and analyzed temporal changes in the pancreatic cancer incidence over the last decade. To this end, an analysis was performed of all pancreatic cancer cases that were registered by the Saudi Cancer Registry between January 2004 and December 2015.

\section{Patients and Methods}

We conducted a retrospective, descriptive epidemiological study of all pancreatic cancer cases diagnosed in Saudi Arabia between January 2004 and December 2015. These data are publicly available and easily accessible through the reports of the Saudi Cancer Registry; as such, no ethical approval was required for this descriptive epidemiological study. The Saudi Cancer Registry is a population-based registry established by the Saudi Ministry of Health in 1994 with an aim to define trends in the incidence and prevalence of different cancer types in Saudi Arabia. It provides statistics on cancer incidence that can be used for planning and effective measurement on early detection and management of cancer. The most recent available dataset from the Saudi Cancer Registry covers through 2015. This study, therefore, explores the epidemiological pattern of pancreatic cancer in Saudi Arabia from 2004 to 2015. Overall, comprehensive reports exist for 13 administrative regions in Saudi Arabia, providing the frequency with percentage of cases, the crude incidence rate (CIR), and the ASIR, stratified by the regions of Saudi Arabia, gender of patients, and years of diagnoses. For this analysis, the CIR was defined as the number of new pancreatic cancer cases occurring in a specific population over a one year period. These crude rates do not take into account variations in age structures from different populations, hampering the comparison of different epidemiological datasets. This is of particular importance in the context of cancer, given the fact that age is a key determinant for the risk of developing cancer. To overcome this, epidemiological studies usually correct for different age structures and use ASIRs as a measure for incidence. This ASIR indicates the incidence rate in 
a specific period, which would be expected in the considered population if the age structure would be similar to that of a predefined standard population. The use of ASIRs allows comparisons of cancer risk between registries, independent of varying age distributions.

Data were analyzed using the Statistical Package for the Social Sciences (SPSS) version 20.0 (IBM Corporation, Armonk, NY, USA). The descriptive analysis of the epidemiological data was performed by calculating the overall percentage, the age-specific incidence rate, the CIR, and the ASIR stratified by gender, region, and year of diagnosis.

The differences in the CIR and ASIR between the year 2004 and the year 2015 were calculated from the reports published by the Saudi Cancer Registry to investigate the pattern of pancreatic cancer among male and female Saudis in different regions. The independent sample $t$-test was used to compare the CIR and ASIR of pancreatic cancer among male and female Saudis, where the units of analyses were CIRs and ASIRs for the 12 years. Furthermore, an analysis of variance test (ANOVA) was used to compare the CIR and ASIR of pancreatic cancer among the different geographical regions in Saudi Arabia. ANOVA test is usually used to compare the means of one independent qualitative variable with one dependent continuous variable. The independent variable should have more than two subgroups. The most important assumptions for conducting the ANOVA test are that the comparison groups must be independent, the dependent variable must be on continuous scale and normally distributed, and homogeneity of variance that can be determined by conducting Levene's test. It is impossible to identify from the $F$-ratio which means differ significantly from other means in the AVOVA test; therefore, the post hoc test should be conducted. However, the post hoc test and Bonferroni correction procedure were used for comparison the specific differences of the CIR and ASIR of pancreatic cancer in different geographical regions of Saudi Arabia.

\section{Results}

\section{Pancreatic Cancer in Saudi Males Total Cases in Saudi Males}

Of the 2338 pancreatic cancer cases registered in Saudi Arabia between January 2004 and December 2015, 1443 were male. Over the 12-year period included in this analysis, a slight increase in the yearly frequency of pancreatic cancer cases was noticed; in 2004, a total of 71 cases were recorded, accounting for $4.9 \%$ of the total amount of pancreatic cancer patients. By 2007, this increased to 127 cases, representing a $3.9 \%$ increase. In the year 2008 , this figure dropped to 100 , after which we observed a steady increase to 175 cases in 2014 (12.1\% of all pancreatic cancer cases in the Saudi Cancer registry). On average, the yearly number of male pancreatic cancer patients over the 12-year period was 120 (Figure 1A).

When stratifying the number of pancreatic cancer patients by age groups, with a class width of 5 years, it becomes clear that the highest number of cases occurred in patients aged 75 years or more $(\mathrm{N}=296,20.5 \%$ of all male pancreatic cancer patients). In this age group, the yearly number of pancreatic cancer cases was 25 . Overall, almost three-quarters (74.3\%) of diagnoses were made in men aged 55 years or more. The overall ASIR from 2004 to 2015 was highest among Saudi males in the age groups $\geq 75,70-74,65-69$, and $60-64$ years at 19.8, 17.6, 14.4, and 9.9 per 100,000, respectively (Figure 1A). Interestingly, the ASIR male-to-female ratio gradually increased with increasing age, from 1.5 in the age group of 35 to 39 years to 1.9 among patients aged 75 years or above (Figure 2).

\section{CIR in Saudi Males}

An analysis of the yearly CIR of pancreatic cancer per 100,000 males indicates a slight increase from 2004 to 2015. In 2004, the CIR was reported at 0.9, increasing to 1.4 in 2007. In 2008, the CIR decreased again to 1.1, after which the CIR gradually increased to 1.7 in 2014 . Over the entire study period, the mean CIR of pancreatic cancer was 1.28 per 100,000 males (95\% confidence interval [CI]: 1.13-1.43). When comparing the CIR of pancreatic cancer among Saudi males and females, an independent sample $t$-test indicated that the CIR was significantly higher among males than females $(p<0.001)$. Overall, the CIR male-to-female ratio from 2004 to 2015 per 100,000 males was 1.6 (Figure 3).

The pancreatic cancer CIR among males varied substantially between the different regions in Saudi Arabia. From 2004 to 2015, the highest overall CIR was reported in Riyadh $(1.5 / 100,000)$, Makkah $(1.5 / 100,000)$, and in the Eastern Region of the Kingdom $(1.5 / 100,000)$. An analysis of variance (ANOVA) proved to be statistically significant for these regions compared with other parts of Saudi Arabia ( $F$ $[12,143]=3.718, p<0.001)$. The regions with the lowest overall CIR were Jizan and Baha 0.5/100,000 and 0.7/100,000, respectively. The CIR male-to-female ratio per region ranged between 1.0 and 2.0, with the exception of the Qassim region, where the male predominance was pronounced, resulting in a CIR male-to-female ratio of 3.3 (Figure 4). 

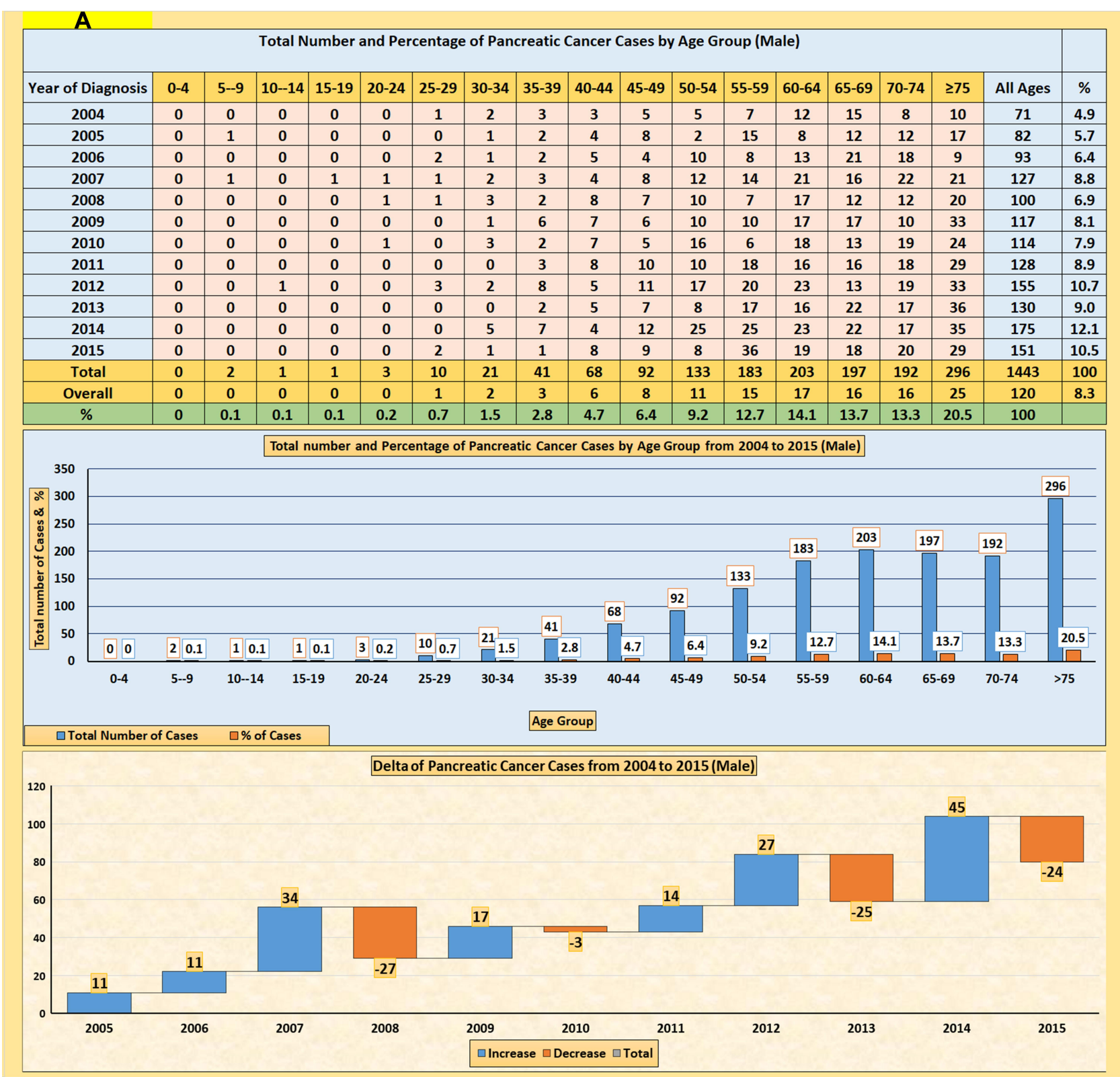

Figure I Continue. 


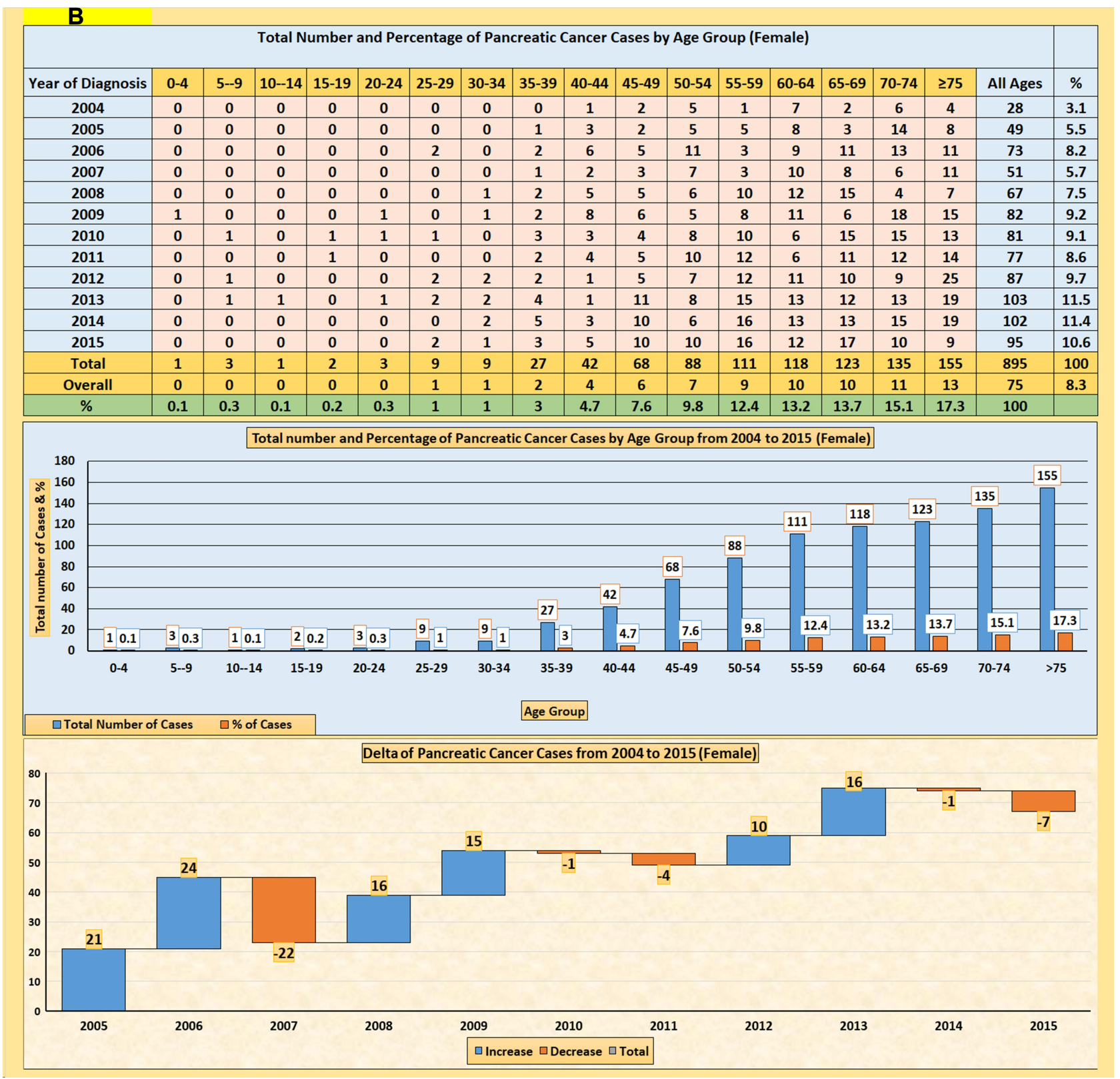

Figure I (A) The total number and percentage of pancreatic cancer cases among male Saudis, with delta values from 2004 to 2015. (B) The total number and percentage of pancreatic cancer cases among female Saudis, with delta values from 2004 to 2015. 


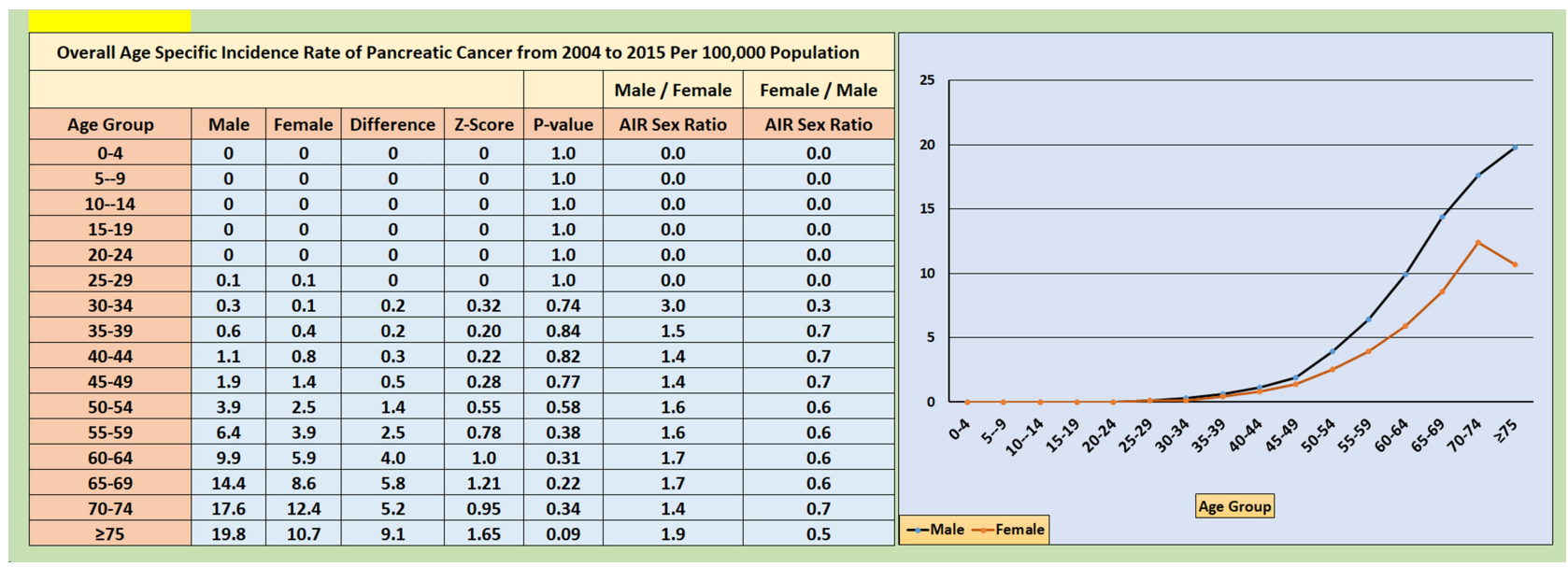

Figure 2 Overall incidence rate (age-specific) of pancreatic cancer cases among both Saudi genders.

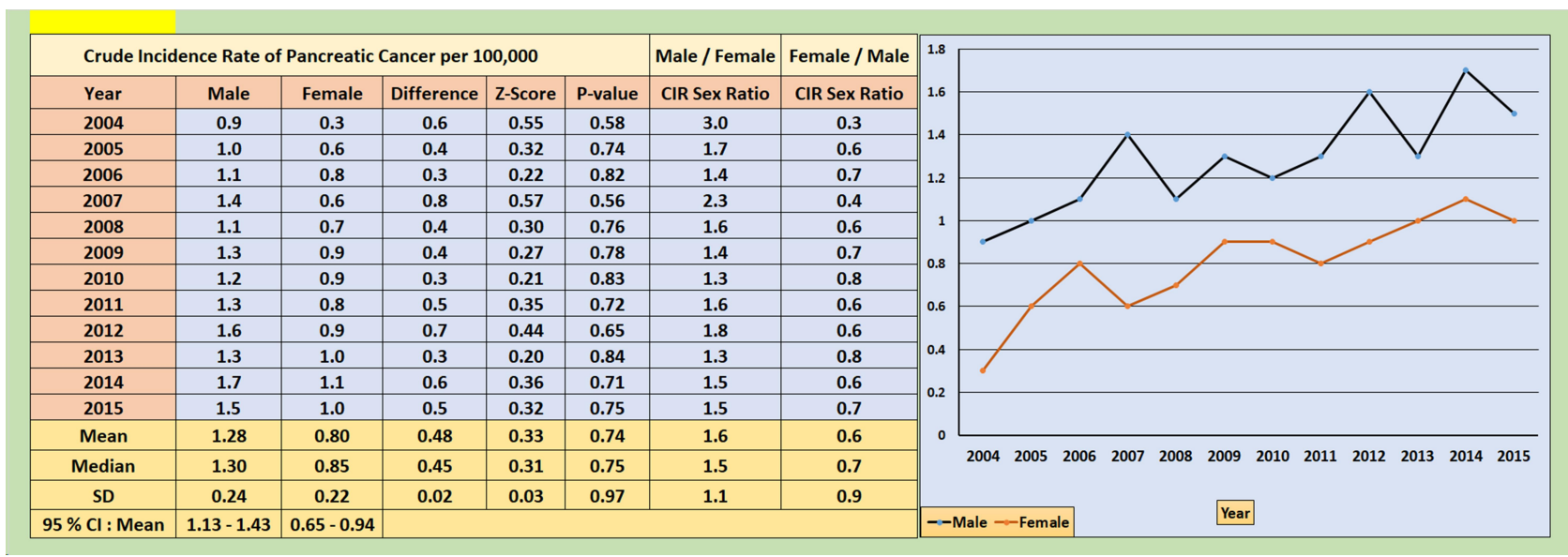

Figure $3 \mathrm{CIR}$ of pancreatic cancer cases among both Saudi genders.

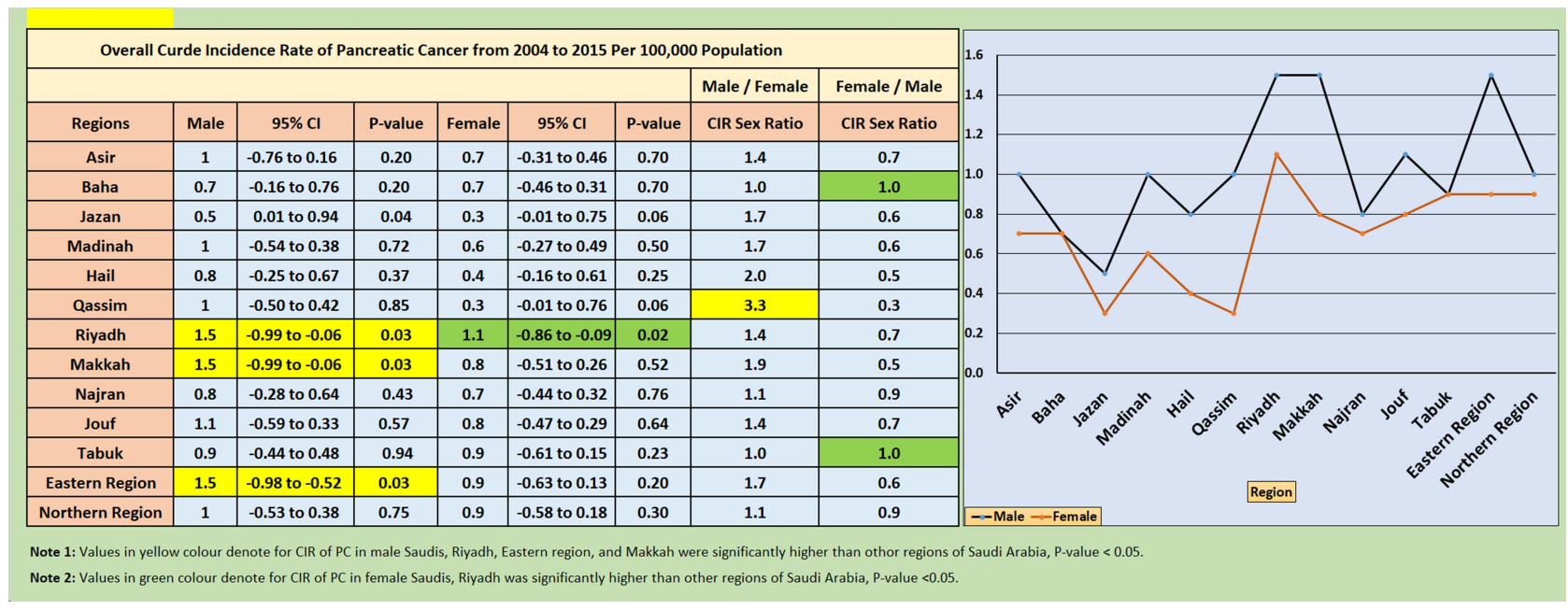

Figure 4 Overall CIR of pancreatic cancer cases among both Saudi genders by region.

Notes: Values in yellow colour denote for CIR of PC in male Saudis, Riyadh, Eastern region, and Makkah were significantly higher than other regions of Saudi Arabia, P-value $<0.05$. Values in green colour denote for CIR of PC in female Saudis, Riyadh was significantly higher than other regions of Saudi Arabia, P-value $<0.05$. 


\section{ASIR in Saudi Males}

Over the 12-year period studied in this analysis, the ASIR for pancreatic cancer among Saudi males was 2.26 (95\% CI: 2.1-2.4). Over this period, the highest ASIR among males was reported in 2007 (2.7) and 2012 (2.6), with the lowest ASIR in the years 2004 (1.7) and 2005 (1.9). The results of the two independent sample t-tests indicated that the ASIR of pancreatic cancer was significantly higher among Saudi males than females $(\mathrm{t}[22]=6.898, \mathrm{p}<0.001)$. Overall, ASIR male-to-female ratio from 2004 to 2015 per 100,000 was 1.6 (Figure 5).

Looking at the male ASIR for pancreatic cancer in function of the geographical region indicated the highest overall ASIR in the Eastern Region (3.2/100,000), Riyadh (3.0/ 100,000), and Tabuk (2.6/100,000). A subsequent ANOVA analysis revealed that this ASIR in these regions was statistically higher compared to other parts of the country $(\mathrm{F}[12,143]$ $=5.534, \mathrm{p}<0.001)$. The lowest incidence among males was seen in Baha, Jizan, and Hail, each with an ASIR of 0.9/ 100,000. The ASIR male-to-female ratio ranged between 1.0 and 1.8 across all the geographical regions, except for Qassim, where this ratio was much higher at 4 (Figure 6).

Looking at the evolution of the ASIR from 2004 to 2015 indicated that the incidence increased most in the northern region $(0.0 / 100,000$ to $3.3 / 100,000)$ and in Tabuk $(0.0 / 100,000$ to $3.1 / 100,000)$, but not statistically significant $p>0.05$. In contrast, the biggest decrease in the male pancreatic cancer ASIR was seen in Hail, where the ASIR decreased from 2.2/ 100,000 in 2004 to $1.2 / 100,000$ in 2015 , but not statistically significant $\mathrm{p}>0.05$ (Figure 7).

\section{Pancreatic Cancer in Saudi Females}

Total Cases in Saudi Females

In total, 895 women were diagnosed with pancreatic cancer in Saudi Arabia between January 2004 and December 2015. Similar to what was seen among males, the number of new cases per year gradually increased over the study period; in 2004 , only 28 cases were registered (3.1\% of the total number of female pancreatic cancer cases), increasing to 82 cases in 2009 and 102 (11.4\%) in 2014. Over the entire period, the average number of pancreatic cancer cases per year was 75 . As in the male cohort, most pancreatic cancer cases were seen in women aged 75 years or more $(\mathrm{N}=155 ; 17.3 \%)$. Overall, women aged $\geq 55$ years accounted for $71.7 \%$ of all pancreatic cancer (Figure 1B). The overall ASIR from 2004 to 2015 was highest among Saudi females in the age groups 70-74, $\geq 75,65-69$, and $60-64$ years at $12.4,10.7,8.6$, and 5.9 per 100,000 , respectively (Figure 2).

\section{CIR in Saudi Females}

The CIR of pancreatic cancer among Saudi females was $0.3 / 100,000$ in 2014, doubling to $0.6 / 100,000$ in 2005 . From 2009 onwards, the CIR remained relatively stable and ranged between 0.9 and 1.1/100,000. On average, the CIR in females over the entire study period was reported at 0.80 (95\% CI: 0.65-0.94) (Figure 3). Looking at the overall CIR in function of the geographical region showed that the pancreatic cancer CIR among Saudi women was highest in the region Riyadh, where the CIR of $1.1 / 100,000$ proved to be significantly higher than the CIR in the other regions (ANOVA tested, $F[12,143]=3.284, p<0.001$ ). The lowest CIR among females was seen for the regions of

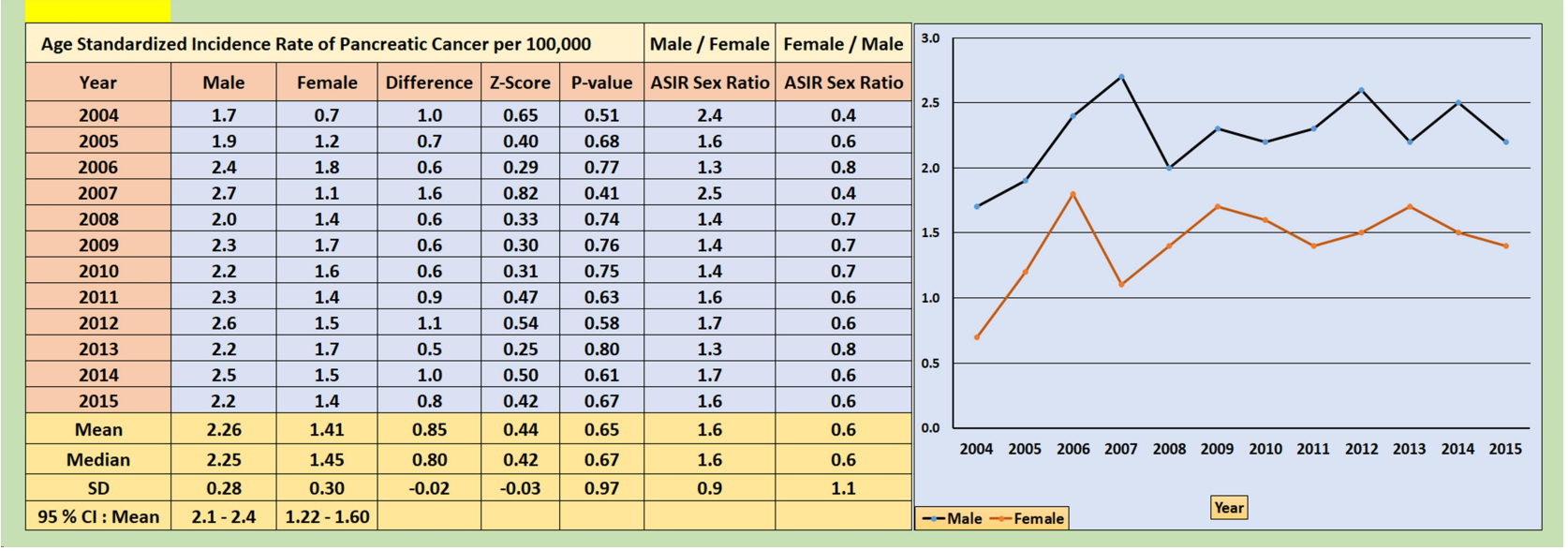

Figure 5 The ASIR of pancreatic cancer cases among both Saudi genders. 


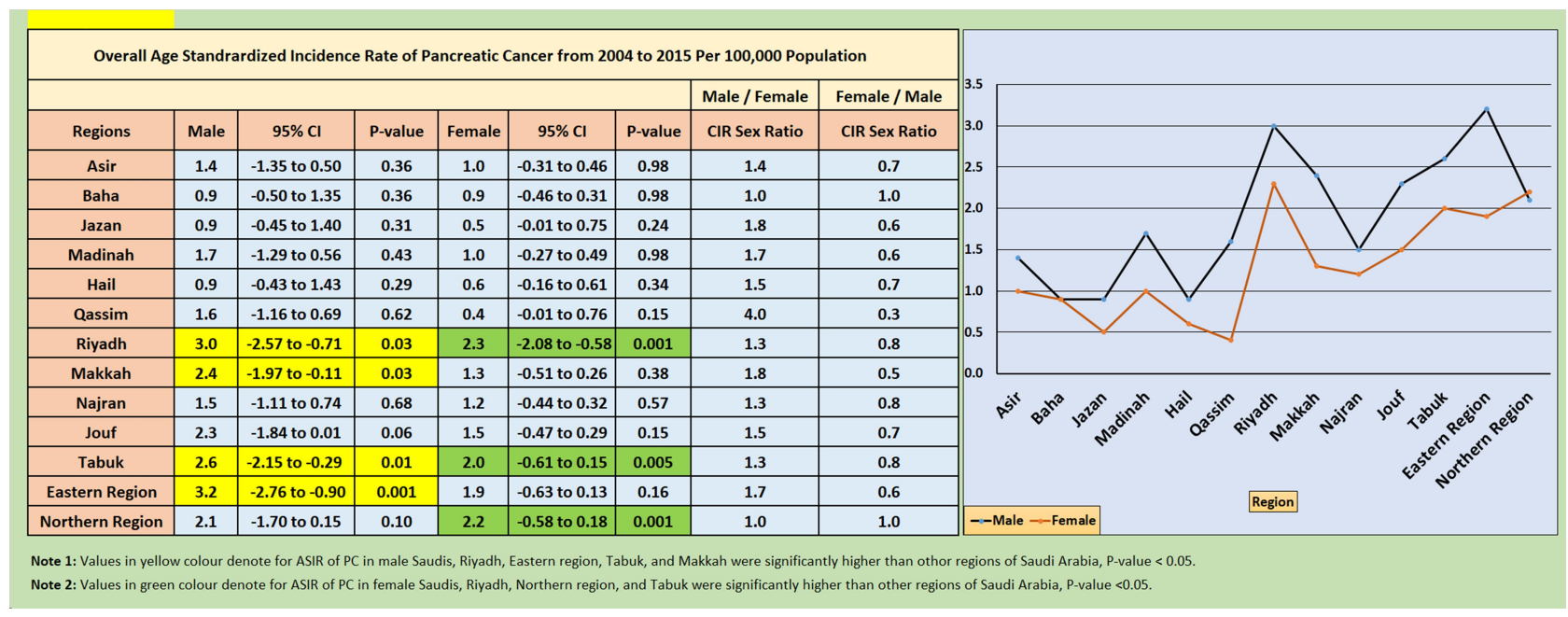

Figure 6 Overall ASIR of pancreatic cancer cases among both Saudi genders by region.

Notes: Values in yellow colour denote for ASIR of PC in male Saudis, Riyadh, Eastern region, Tabuk, and Makkah were significantly higher than other regions of Saudi Arabia, P-value $<0.05$. Values in green colour denote for ASIR of PC in female Saudis, Riyadh, Northern region, and Tabuk were significantly higher than other regions of Saudi Arabia, P-value $<0.05$.

\begin{tabular}{|c|c|c|c|c|c|c|c|c|c|c|c|c|c|c|c|}
\hline \multirow[b]{2}{*}{ Region } & \multicolumn{15}{|c|}{ The differences in the CIR and ASIR between the year 2004 and the year 2015} \\
\hline & \multirow{3}{*}{ Male } & & \multirow{2}{*}{$\begin{array}{c}2004 \\
0.6\end{array}$} & \multirow{2}{*}{$\begin{array}{c}2015 \\
1.0\end{array}$} & \multirow{2}{*}{\begin{tabular}{|c|} 
Difference \\
0.4
\end{tabular}} & \multirow{2}{*}{\begin{tabular}{|c|} 
Z-Score \\
0.32
\end{tabular}} & \multirow{2}{*}{\begin{tabular}{|c|} 
P-value \\
0.74
\end{tabular}} & \multirow{5}{*}{$\begin{array}{l}\text { Region } \\
\text { Riyadh }\end{array}$} & \multicolumn{2}{|c|}{ Sex } & \multirow{2}{*}{$\begin{array}{c}2004 \\
0.9\end{array}$} & \multirow{2}{*}{$\begin{array}{c}2015 \\
1.7\end{array}$} & \multirow{2}{*}{$\begin{array}{c}\text { Difference } \\
0.8\end{array}$} & \multirow{2}{*}{\begin{tabular}{|c|} 
Z-Score \\
0.5
\end{tabular}} & \multirow{2}{*}{\begin{tabular}{|c|} 
P-value \\
0.61
\end{tabular}} \\
\hline \multirow{4}{*}{ Asir } & & CIR & & & & & & & Male & CIR & & & & & \\
\hline & & ASIR & 1.1 & 0.9 & -0.2 & -0.14 & 0.88 & & IViale & ASIR & 2.2 & 2.8 & 0.6 & 0.27 & 0.78 \\
\hline & \multirow{2}{*}{ Female } & CIR & 0.3 & 0.3 & 0.0 & 0.0 & 1.0 & & \multirow{2}{*}{ Female } & CIR & 0.7 & 1.6 & 0.9 & 0.59 & 0.55 \\
\hline & & ASIR & 0.3 & 0.4 & 0.1 & 0.12 & 0.90 & & & ASIR & 1.7 & 2.7 & 1.0 & 0.48 & 0.63 \\
\hline \multirow{4}{*}{ Baha } & \multirow{2}{*}{ Male } & CIR & 0.7 & 0.0 & -0.7 & -0.84 & 0.40 & \multirow{4}{*}{ Makkah } & \multirow{2}{*}{ Male } & CIR & 1.4 & 1.5 & 0.1 & 0.06 & 0.95 \\
\hline & & ASIR & 0.9 & 0.0 & -0.9 & -0.95 & 0.34 & & & ASIR & 2.5 & 1.9 & -0.6 & -0.29 & 0.77 \\
\hline & \multirow{2}{*}{ Female } & CIR & 0.6 & 0.5 & -0.1 & -0.1 & 0.92 & & \multirow{2}{*}{ Female } & CIR & 0.4 & 1.2 & 0.8 & 0.63 & 0.52 \\
\hline & & ASIR & 0.7 & 0.6 & -0.1 & -0.09 & 0.99 & & & ASIR & 0.8 & 1.6 & 0.8 & 0.52 & 0.60 \\
\hline \multirow{4}{*}{ Jazan } & $M a l$ & CIR & 0.2 & 0.8 & 0.6 & 0.6 & 0.54 & & Malo & CIR & 0.6 & 0.9 & 0.3 & 0.24 & 0.81 \\
\hline & Tiale & ASIR & 2.0 & 1.1 & -0.9 & -0.51 & 0.61 & & IViale & ASIR & 1.7 & 1.6 & -0.1 & -0.06 & 0.95 \\
\hline & & CIR & 0.0 & 0.5 & 0.5 & 0.71 & 0.47 & Najran & & CIR & 0.0 & 1.4 & 1.4 & 1.18 & 0.23 \\
\hline & remale & ASIR & 0.0 & 0.6 & 0.6 & 0.77 & 0.44 & & remale & ASIR & 0.0 & 1.8 & 1.8 & 1.34 & 0.18 \\
\hline & $M$ & CIR & 0.4 & 0.9 & 0.5 & 0.44 & 0.65 & & & CIR & 1.3 & 2.7 & 1.4 & 0.7 & 0.48 \\
\hline Madinah & |Viale & ASIR & 0.5 & 1.3 & 0.8 & 0.6 & 0.54 & & IViale & ASIR & 3.4 & 4.7 & 1.3 & 0.46 & 0.64 \\
\hline IViauinan & $50 m a t$ & CIR & 0.0 & 0.2 & 0.2 & 0.45 & 0.65 & Jout & Tomola & CIR & 0.0 & 0.0 & 0.0 & 0.0 & 1.0 \\
\hline & Femaie & ASIR & 0.0 & 0.3 & 0.3 & 0.55 & 0.58 & & Female & ASIR & 0.0 & 0.0 & 0.0 & 0.0 & 1.0 \\
\hline & Malo & CIR & 1.8 & 0.8 & -1.0 & -0.62 & 0.53 & & Malo & CIR & 0.0 & 1.7 & 1.7 & 1.30 & 0.19 \\
\hline Hoil & |iviale & ASIR & 2.2 & 1.2 & -1.0 & -0.54 & 0.58 & Tahus & IViale & ASIR & 0.0 & 3.1 & 3.1 & 1.76 & 0.07 \\
\hline Hall & Femal & CIR & 0.0 & 0.4 & 0.4 & 0.63 & 0.53 & labuk & Femalo & CIR & 0.3 & 1.8 & 1.5 & 1.04 & 0.29 \\
\hline & Female & ASIR & 0.0 & 0.5 & 0.5 & 0.71 & 0.47 & & Fernale & ASIR & 0.9 & 2.5 & 1.6 & 0.87 & 0.38 \\
\hline & Malo & CIR & 0.7 & 0.4 & -0.3 & -0.29 & 0.77 & & Malo & CIR & 0.8 & 2.3 & 1.5 & 0.85 & 0.39 \\
\hline $0 ?$ & IViale & ASIR & 0.3 & 0.5 & 0.2 & 0.22 & 0.82 & Eastern & fiviale & ASIR & 1.9 & 3.9 & 2.0 & 0.83 & 0.40 \\
\hline Qdassimin & Eemale & CIR & 0.0 & 0.4 & 0.4 & 0.63 & 0.53 & Region & Female & CIR & 0.3 & 0.9 & 0.6 & 0.55 & 0.58 \\
\hline & remale & ASIR & 0.0 & 0.5 & 0.5 & 0.71 & 0.47 & & pernale & ASIR & 0.7 & 1.4 & 0.7 & 0.48 & 0.63 \\
\hline & & & & & & & & & Ma & CIR & 0.0 & 2.1 & 2.1 & 1.45 & 0.14 \\
\hline & & & & & & & & Northern & Jiviare & ASIR & 0.0 & 3.3 & 3.3 & 1.82 & 0.06 \\
\hline & & & & & & & & Region & Female & CIR & 0.3 & 0.7 & 0.4 & 0.4 & 0.68 \\
\hline & & & & & & & & & Female & ASIR & 0.9 & 0.9 & 0.0 & 0.0 & 1.0 \\
\hline
\end{tabular}

Note 1: Values in bold denote the differences in the CIRs and ASIRs of pancreatic cancer cases between 2004 and 2015 in different regions of Saudi Arabia.

Note 2: P-values > 0.05 denote there are no significant differences in the CIRs and ASIRs of pancreatic cancer cases between 2004 and 2015 in different regions of Saudi Arabia.

Figure 7 The differences in the CIR and ASIR of pancreatic cancer in Saudi Arabia.

Notes: Values in bold denote the differences in the CIRs and ASIRs of pancreatic cancer cases between 2004 and 2015 in different regions of Saudi Arabia. P-values $>0.05$ denote there are no significant differences in the CIRs and ASIRs of pancreatic cancer cases between 2004 and 2015 in different regions of Saudi Arabia. 
Jizan and Qassim, each with a CIR of 0.3/100,000, and Hail, where the CIR was 0.4/100,000 (Figure 4).

\section{ASIR in Saudi Females}

Overall, the ASIR of pancreatic cancer among Saudi women was $1.41 / 100,000$ (95\% CI: 1.22-1.60). The lowest ASIR was seen in 2004 at $0.7 / 100,000$. This rapidly increased to $1.2 / 100,000$ in 2005 to peak at $1.8 / 100,000$ in the year 2006. In 2007, the ASIR among females dropped back to $1.1 / 100,000$, after which it remained relatively stable between 1.4/ 100,000 and 1.7/100,000 from 2008 to 2015 (Figure 5).

The regions with the highest overall ASIR for pancreatic cancer in females were Riyadh, the northern region of Saudi Arabia, and the Tabuk region with an ASIR of 2.3, 2.2, and 2.0 per 100,000, respectively. The ANOVA proved to be statistically significant for these regions compared with other parts of Saudi Arabia (F[12,143] =5.784, $\mathrm{p}<0.001)$. The lowest overall ASIR among females was seen in the regions of Qassim, Jizan, and Hail, where the overall ASIR was reported at 0.4, 0.5, and 0.6 per 100,000, respectively (Figure 6).

The most significant temporal changes over the 12-year study period in women were seen in the region of Najran, where the ASIR increased from 0.0/100,000 in 2004 to $1.8 / 100,000$ in 2015, and in the region of Tabuk, where the ASIR increased from 0.9 to $2.5 / 100,000$ over the study period, but not statistically significant $p>0.05$. In the other regions, the incidence of pancreatic cancer in females was fairly similar in 2004 and 2015 (Figure 7).

\section{Summary of Saudi Data and Comparison with Worldwide Data}

The overall epidemiological analysis on the incidence of pancreatic cancer in Saudi Arabia and the gender, age, geographical, and temporal distributions are summarized in Figure 8. These can be compared to the worldwide ASIR as shown in Figure 9 and the age-standardized mortality rate (ASMR) as shown in Figure 10. In 2018, the International Agency for Research on Cancer (IARC) estimated that the ASIR for pancreatic cancer in Saudi Arabia among both sexes of all ages was 2.2 per 100,000 individuals (Figure 9). Based on the published data from the GLOBOCAN, compared to the global ASMR of pancreatic cancer, the mortality of pancreatic cancer in Saudi Arabia is low at only 2.2/ 100,000 . This is also lower than found in most neighboring countries. The highest ASMR for pancreatic

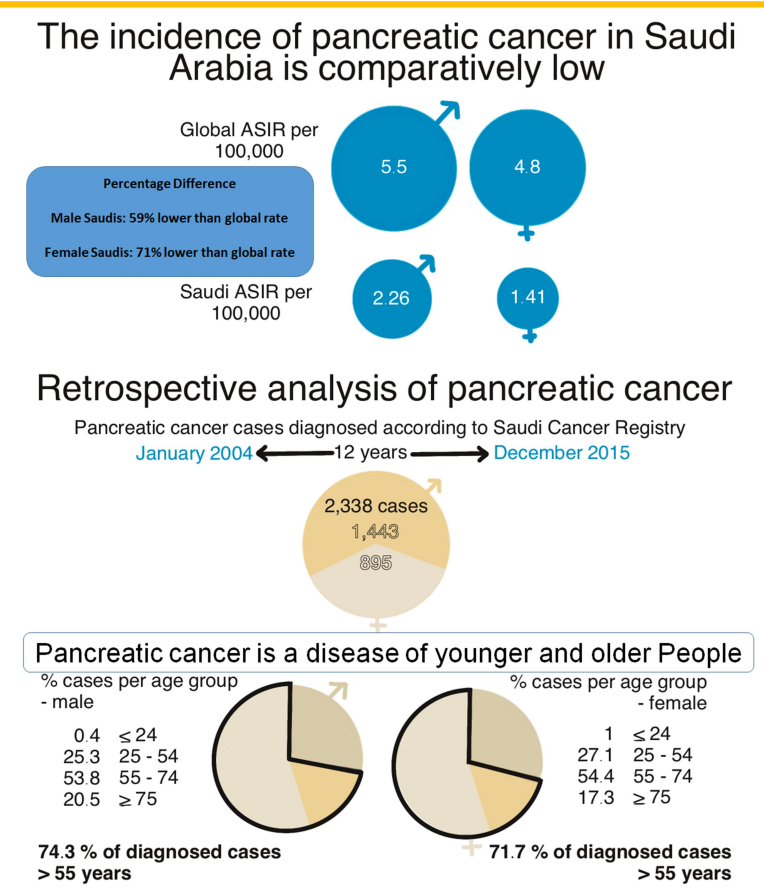

Pancreatic cancer CIR varies substancially between regions and is overall higher in male Saudis

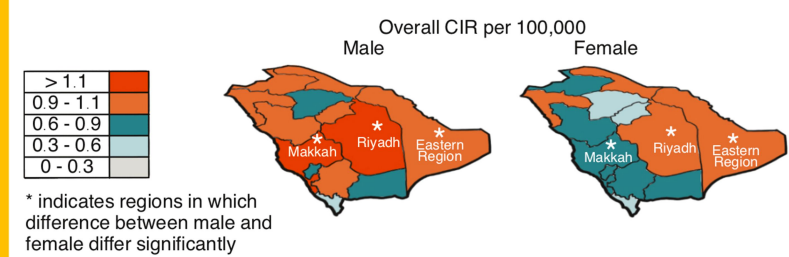
emale differ

Pancreatic cancer ASIR varies substancially between regions and is overall higher in male

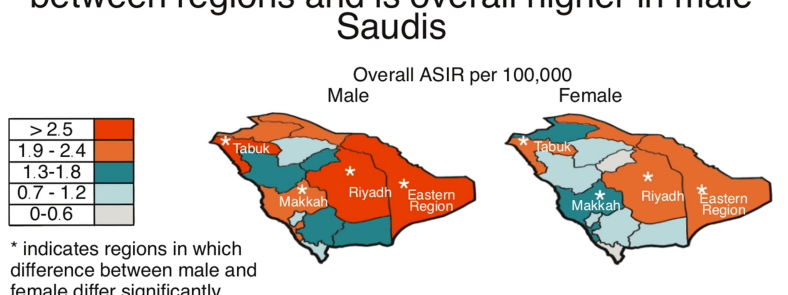

Factors influencing pancreatic incidence

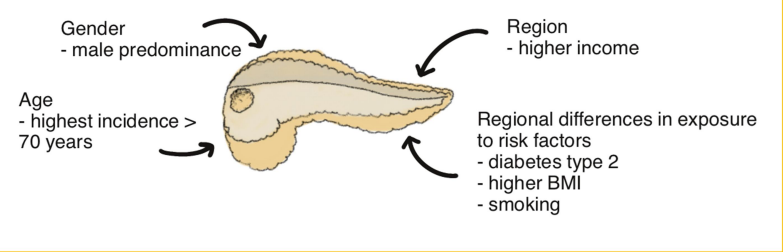

Figure 8 Summary results of pancreatic cancer in Saudi Arabia.

cancer in the region was reported in Israel (7.8/ 100,000), followed by the United Arab Emirates (4.4/ 100,000), Lebanon (3.7/100,000), Jordan (3.7/100,000), Kuwait (3.7/100,000), Syria (3.3/100,000), and Oman (3.0/100,000). 


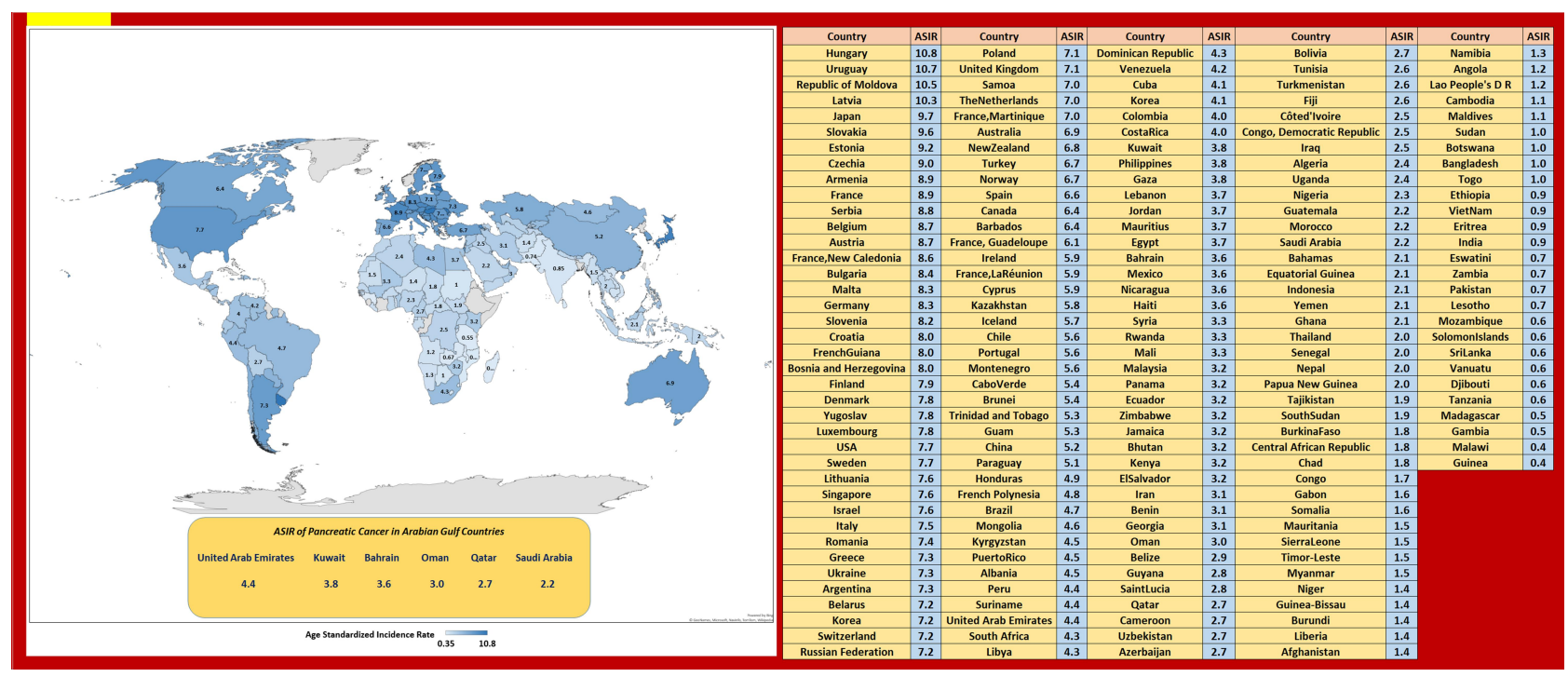

Figure 9 ASIR of pancreatic cancer (world) in 2018, all ages, both genders.

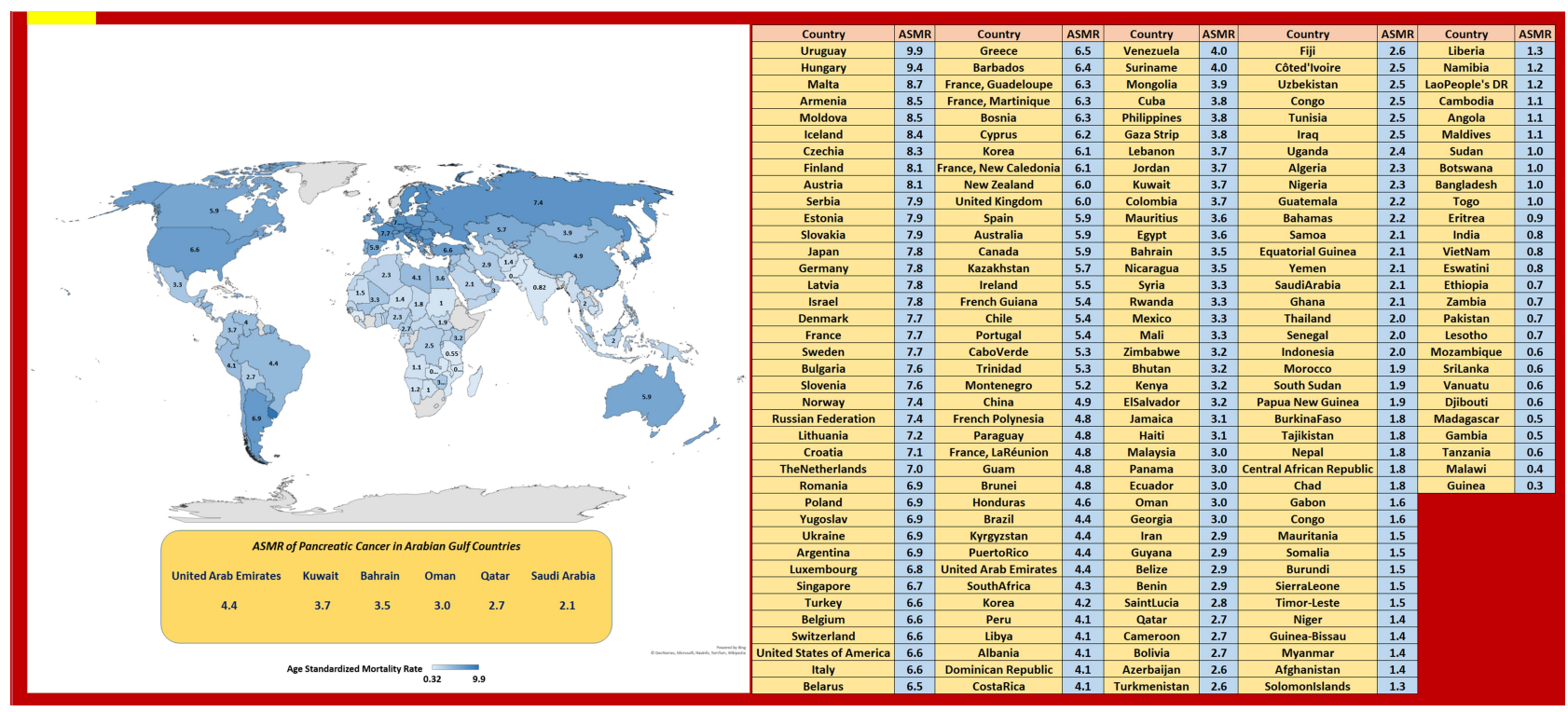

Figure 10 ASMR of pancreatic cancer (world) in 2018, all ages, both genders.

\section{Discussion}

Pancreatic cancer remains a devastating cancer type with a poor prognosis. A required initial analysis that may lead to improved care for patients with pancreatic cancer consists of a thorough understanding of the epidemiological data. In this respect, this study provides the first detailed epidemiological analysis on the incidence of pancreatic cancer in Saudi Arabia and gives insights into the gender, age, geographical, and temporal distributions of this cancer type in the Kingdom.

From January 2004 to December 2015, a total of 2338 cases of pancreatic cancer were registered, consisting of 1443 male and 895 female patients. The CIR of pancreatic cancer was $1.28 / 100,000$ among males and $0.80 / 100,000$ in females, with an ASIR of 2.26 and 1.41/100,000 for males and females, respectively. As such, this analysis confirmed the male predominance of pancreatic cancer with a higher ASIR and CIR among males than females, with a male-tofemale ratio of 1.6 for both the ASIR and the CIR. However, the exact reasons for this male predominance are largely unclear. With the available data, it is difficult to say whether women are actually less prone to developing pancreatic cancer or whether a different exposure to risk factors for pancreatic cancer explains this gender difference. In 2018, the International Agency for Research on Cancer (IARC) 
estimated that the ASIR for pancreatic cancer in Saudi Arabia among both sexes of all ages was 2.2 per 100,000 individuals. ${ }^{4}$ Also, in this dataset, the male predominance of pancreatic cancer was observed with an ASIR of 2.7/100,000 in males and 1.6/100,000 in females. As such, these GLOBOCAN figures are slightly higher than the ASIR extracted from the Saudi Cancer Registry $(2.26 / 100,000$ and 1.41/100,000 for males and females, respectively).

Both in males and females, the age-specific incidence rate of pancreatic cancer increased with increasing age, with the highest incidence in patients aged 70 years or more. This result is similar to worldwide data that identifies the median age of patients with pancreatic cancer as 70 years. Early onset pancreatic cancer is considered when patients are younger than 50 years and this occurs in around $10 \%$ of cases worldwide. ${ }^{9}$ It is important to consider patients with early onset pancreatic cancer, as they may be able to undergo more intensive therapy due to their better overall health. ${ }^{9}$

Overall, the incidence of pancreatic cancer in Saudi Arabia is low compared to the global incidence of this cancer type. In fact, GLOBOCAN data for 2018 indicate a yearly ASIR for pancreatic cancer of 5.5/100,000 among males and of 4.8/100,000 in females. Looking at the incidence of pancreatic cancer in 2018 on a global level confirms the fact that pancreatic cancer is more frequently seen in higher-income regions. In fact, according to GLOBOCAN data, the highest pancreatic cancer incidence in 2018 was reported in Europe and North America, with an ASIR of 7.7 and 7.6/100,000, while the lowest ASIR for pancreatic cancer was found in Africa $(2.2 / 100,000)$. This situation was similar for males and females. ${ }^{4}$ In 2018, the highest overall pancreatic cancer ASIR was in Hungary $(10.8 / 100,000)$. Only looking at the incidence among males, the highest incidence was reported in Latvia $(15.3 / 100,000)$. The highest ASIR for pancreatic cancer in women was seen in the United Arab Emirates $(10.1 / 100,000)$. It is most likely that this large variation in pancreatic cancer incidence around the globe is related to different exposure to risk factors for this cancer type.

Comparing the pancreatic cancer ASIR in Saudi males to other countries on the Arabian Peninsula reveals a reasonably similar picture with an ASIR of 2.0/100,000 in the United Arab Emirates and 1.8/100,000 in Qatar. In contrast, compared to other countries in the Middle-East, the ASIR for pancreatic cancer in Saudi males is 2.7 lower, with an ASIR of 5.1, 4.5, 3.5, and 3.0 per 100,000 in Jordan, Lebanon, Iran, and Iraq, respectively. ${ }^{4}$
Looking at the pancreatic cancer incidence among females in the Middle-East reveals some important differences. In fact, the female pancreatic cancer incidence in Saudi Arabia is 1.6 , the lowest in the entire region. In Iraq, the female pancreatic cancer ASIR was set at 2.1/100,000, while in Oman, Jordan, Iran, and Lebanon, the pancreatic cancer incidence was even higher at 2.3, 2.4, 2.6, and 2.8 per 100,000 , respectively. However, the highest female pancreatic cancer ASIR in the region was reported in the United Arab Emirates, where the ASIR for pancreatic cancer among women was more than sevenfold higher than in Saudi Arabia (ASIR: 10.1/100,000). In fact, this ASIR for pancreatic cancer among women in the United Arab Emirates was the highest incidence worldwide. ${ }^{4}$ These differences in incidence are reflected in different mortality rates. In 2018, pancreatic cancer was associated with a global ASMR of 4.4 per $100,000 .{ }^{4}$ Also, in this respect, significant regional variation is seen. The highest ASMR was seen in Uruguay $(9.9 / 100,000)$, while Guinea was characterized by the lowest ASMR $(0.32 / 100,000) .{ }^{4}$ Therefore, the mortality of pancreatic cancer in Saudi Arabia is low at only 2.2/100,000. This is lower than most of the neighboring countries where the highest ASMR for pancreatic cancer in the region was in Israel (7.8/100,000), followed by the United Arab Emirates (4.4/ 100,000), Lebanon $(3.7 / 100,000)$, Jordan $(3.7 / 100,000)$, Kuwait $(3.7 / 100,000)$, Syria $(3.3 / 100,000)$, and Oman $(3.0 / 100,000)$.

Also, within Saudi Arabia, significant regional variations were seen with the male pancreatic cancer ASIR being significantly higher in the Eastern Region (3.2/ 100,000), Riyadh (3.0/100,000), and Tabuk (2.6/ 100,000). Similarly, among females, the ASIR was also significantly higher in the regions of Riyadh $(2.3 / 100,000)$ and Tabuk $(2.0 / 100,000)$, while in this cohort, also the northern region $(2.2 / 100,000)$ proved to have a significantly higher pancreatic cancer incidence.

The most prominent adjustable risk factor for pancreatic cancer consists of cigarette smoking. Large studies have indicated that current smokers have a $70-75 \%$ increased risk of developing pancreatic cancer compared to never-smokers. ${ }^{10,11}$ Importantly, this increased risk is maintained in former smokers, with ever-smokers having a 20\% higher risk for pancreatic cancer compared to never-smokers. ${ }^{10}$ This increased risk in ever-smokers is particularly pronounced during the first 5 years after they stop smoking. After that time, the risk for pancreatic cancer drops back to comparable levels to never- 
smokers. ${ }^{11}$ In 2015, Moradi-Lakeh et al reported the results of a cross-sectional study assessing tobacco consumption in Saudi Arabia. For this analysis, a survey was performed among 10,735 individuals aged 15 years or above. ${ }^{12}$ This analysis revealed an overall current smoking prevalence of $12.2 \%$ (21.5\% in men, $1.1 \%$ among female). Interestingly, the highest prevalence of current tobacco use was reported in the Tabuk region (18.5\%), and this region was also characterized as the region with the highest prevalence of heavy smoking (11.7\%). In addition to this, the eastern regions of Saudi Arabia were characterized by the highest prevalence of daily shisha smoking $(5.31 \%$ to $6.82 \%){ }^{12}$ This higher prevalence of tobacco use in Tabuk and the eastern part of the country may contribute to the high overall ASIR of pancreatic cancer in these regions among Saudi males from 2004 to 2015.

Part of the explanation for the particularly high pancreatic cancer incidence among women in the United Arab Emirates could be that it also has one of the highest prevalence rates of type 2 diabetes in the world. ${ }^{13}$ Many epidemiological studies established a strong association between pancreatic cancer and diabetes. ${ }^{14,15}$ In light of the current obesity pandemic in many different regions of the world, it is important to underscore that obesity is also considered to be a strong promotor of diabetes mellitus. Apart from its effect through the development of diabetes, a higher body mass index on itself was also found to increase the risk of pancreatic cancer. ${ }^{8,16,17}$ As such, the ever-increasing incidence of obesity, especially in higher-income countries, is believed to play an important role in the increasing incidence of pancreatic cancer seen in these regions. The prevalence of diabetes mellitus in Saudi Arabia is rising and has been higher in urban regions than in rural parts of the country, with the highest diabetes prevalence seen in Riyadh, as confirmed by a national survey by Saudi Ministry of Health in $2013 .{ }^{18,19}$ In that analysis, the prevalence of diabetes mellitus in the region of Riyadh was found to be 20 times higher than Jizan. ${ }^{20}$ It is very likely that the high prevalence of diabetes mellitus has contributed to the fact that Riyadh also had the highest overall ASIR of pancreatic cancer from 2004 to 2015. As such, this suggests that a high prevalence of diabetes may be associated with higher ASIR of pancreatic cancer in males and females residing in Riyadh.

The ASIR for pancreatic cancer gradually increased from 2004 to 2015, with an increase from 1.7/100,000 to
$2.2 / 100,000$ in males and from $0.7 / 100,000$ to $1.4 / 100,000$ in females. In absolute numbers, this gradual increase is perhaps more obvious. In males, the yearly number of pancreatic cancer diagnoses more than doubled from 71 in 2004 to 151 in 2015. Overall, approximately a third of all pancreatic cancer diagnoses among males were registered in the last three years of the analysis (the years 2013, 2014, and 2015, accounting for 31.6\% of all male pancreatic cancer cases). A similar picture was seen among women, where the yearly number of cases more than tripled from only 28 in 2004 to 95 in 2015 . Also, among women, a third of cases were registered in the years 2013, 2014, and 2015 (33,5\%). This observation is in line with the steady increase in the incidence of pancreatic cancer seen in other parts of the world. For example, an analysis of data in the US from 1973 to 2014 has revealed a steady increase in the pancreatic cancer ASIR of $1.03 \%$ per year. ${ }^{21}$ Again, a clear explanation for this steadily increasing incidence is difficult to give and is likely to be multifactorial. Part of the explanation could be the rapid growth in the rate of obesity in Saudi Arabia. In fact, over the last decades, obesity became a major public health problem in Saudi Arabia. ${ }^{22}$ Simultaneously, the incidence of diabetes has been rising in Saudi Arabia over the last decades.

Our study has several limitations that may have influenced the results. The descriptive nature of the study, without a comparison group, means a statistical association between variables cannot be tested. ${ }^{23}$ The true incidence of pancreatic cancer may have been underestimated from the Saudi Cancer Registry, which as a pathological database does not record patients without a pathological diagnosis. There was an absence of survival data in the registry, so the overall mortality rates of pancreatic cancer in different regions of Saudi Arabia were not calculated in this study. Despite these limitations, with the geographic distribution of pancreatic cancer among male and female Saudis being unavailable, except for the Saudi Cancer Registry reports, we intended to clarify the real patterns of pancreatic cancer in different regions from 2004 until 2015. Therefore, these study findings provide highly relevant information for researchers and health-care policy makers in Saudi Arabia.

\section{Conclusions}

This study revealed a slight increase of the CIR and ASIR of pancreatic cancer among males and females of the Saudi population. Eastern region, Riyadh, and Tabuk had 
the highest overall ASIRs of pancreatic cancer among males, Riyadh, Northern region, and Tabuk among Saudi females, while the Jazan recorded the lowest rate among male and female Saudis. The rates of pancreatic cancer in Saudi Arabia were significantly higher among males compared with female Saudis. Further analytical studies are needed to identify the potential risk factors for pancreatic cancer among the Saudi population.

\section{Disclosure}

The authors report no conflicts of interest in this work.

\section{References}

1. Ilic M, Ilic I. Epidemiology of pancreatic cancer. World J Gastroenterol. 2016;22:9694-9705. doi:10.3748/wjg.v22.i44.9694

2. Strobel O, Neoptolemos J, Jäger D, Büchler MW. Optimizing the outcomes of pancreatic cancer surgery. Nat Rev Clin Oncol. 2019;16:11-26. doi:10.1038/s41571-018-0112-1

3. Bray F, Ferlay J, Soerjomataram I, et al. Global cancer statistics 2018: GLOBOCAN estimates of incidence and mortality worldwide for 36 cancers in 185 countries. CA Cancer J Clin. 2018;68:394-424. doi: $10.3322 /$ caac. 21492

4. GLOBOCAN. CLOBOCAN cancer Fact Sheet pancreatic cancer; 2019. Available from: https://gco.iarc.fr/today/data/factsheets/cancers/ 13-Pancreas-fact-sheet.pdf. Accessed January 12, 2021.

5. Pourshams A, Sepanlou SG, Ikuta KS; Collaborators, G. B. D. P. C. The global, regional, and national burden of pancreatic cancer and its attributable risk factors in 195 countries and territories, 1990-2017: a systematic analysis for the Global Burden of Disease Study 2017 Lancet Gastroenterol Hepatol. 2019;4:934-947. doi:10.1016/S24681253(19)30347-4.

6. Wong MCS, Jiang JY, Liang M, et al. Global temporal patterns of pancreatic cancer and association with socioeconomic development. Sci Rep. 2017;7:3165. doi:10.1038/s41598-017-02997-2

7. Midha S, Chawla S, Garg PK. Modifiable and non-modifiable risk factors for pancreatic cancer: a review. Cancer Lett. 2016;381:269-277. doi:10.1016/j.canlet.2016.07.022

8. Arslan AA, Helzlsouer KJ, Kooperberg C, et al. Anthropometric measures, body mass index, and pancreatic cancer: a pooled analysis from the Pancreatic Cancer Cohort Consortium (PanScan). Arch Intern Med. 2010;170:791-802. doi:10.1001/archinternmed.2010.63

9. Primavesi F, Stättner S, Schlick K, et al. Pancreatic cancer in young adults: changes, challenges, and solutions. Onco Targets Ther. 2019;12:3387-3400. doi:10.2147/ott.S176700
10. Iodice S, Gandini S, Maisonneuve P, Lowenfels AB. Tobacco and the risk of pancreatic cancer: a review and meta-analysis. Langenbecks Arch Surg. 2008;393:535-545. doi:10.1007/s00423-007-0266-2

11. Vrieling A, Bueno-de-Mesquita HB, Boshuizen HC, et al. Cigarette smoking, environmental tobacco smoke exposure and pancreatic cancer risk in the European Prospective Investigation into Cancer and Nutrition. Int $J$ Cancer. 2010;126:2394-2403. doi:10.1002/ ijc. 24907

12. Moradi-Lakeh M, El Bcheraoui C, Tuffaha M, et al. Tobacco consumption in the Kingdom of Saudi Arabia, 2013: findings from a national survey. BMC Public Health. 2015;15:611. doi:10.1186/ s12889-015-1902-3

13. Shaw JE, Sicree RA, Zimmet PZ. Global estimates of the prevalence of diabetes for 2010 and 2030. Diabetes Res Clin Pract. 2010;87:4-14. doi:10.1016/j.diabres.2009.10.007

14. Stevens RJ, Roddam AW, Beral V. Pancreatic cancer in type 1 and young-onset diabetes: systematic review and meta-analysis. $\mathrm{Br}$ J Cancer. 2007;96:507-509. doi:10.1038/sj.bjc.6603571

15. Huxley R, Ansary-Moghaddam A, Berrington de González A, Barzi F, Woodward M. Type-II diabetes and pancreatic cancer: a meta-analysis of 36 studies. Br J Cancer. 2005;92:2076-2083. doi:10.1038/sj.bjc.6602619

16. Zhou B, Wu D, Liu H, et al. Obesity and pancreatic cancer: an update of epidemiological evidence and molecular mechanisms. Pancreatology. 2019;19:941-950. doi:10.1016/j.pan.2019.08.008

17. Michaud DS. Obesity and Pancreatic Cancer. Recent Results Cancer Res. 2016;208:95-105. doi:10.1007/978-3-319-42542-9_6

18. Al-Nozha M, Al-Maatouq MA, Al-Mazrou YY, et al. Diabetes mellitus in Saudi Arabia. Saudi Med J. 2004;25:1603-1610.

19. Alotaibi A, Perry L, Gholizadeh L, Al-Ganmi A. Incidence and prevalence rates of diabetes mellitus in Saudi Arabia: an overview. $J$ Epidemiol Glob Health. 2017;7:211-218. doi:10.1016/j. jegh.2017.10.001

20. Alghamdi IG, Alghamdi MS. The incidence rate of liver cancer in saudi arabia: an observational descriptive epidemiological analysis of data from the Saudi Cancer Registry (2004-2014). Cancer Manag Res. 2020;12:1101-1111. doi:10.2147/cmar.S232600

21. Saad AM, Turk T, Al-Husseini MJ, Abdel-Rahman O. Trends in pancreatic adenocarcinoma incidence and mortality in the United States in the last four decades; a SEER-based study. BMC Cancer. 2018;18:688. doi:10.1186/s12885-018-4610-4

22. DeNicola E, Aburizaiza OS, Siddique A, Khwaja H, Carpenter DO. Obesity and public health in the Kingdom of Saudi Arabia. Rev Environ Health. 2015;30:191-205. doi:10.1515/reveh-2015-0008

23. Aggarwal R, Ranganathan P. Study designs: part 2 - descriptive studies. Perspect Clin Res. 2019;10:34-36. doi:10.4103/picr. PICR_154_18
Clinical and Experimental Gastroenterology

\section{Publish your work in this journal}

Clinical and Experimental Gastroenterology is an international, peerreviewed, open access, online journal publishing original research, reports, editorials, reviews and commentaries on all aspects of gastroenterology in the clinic and laboratory. This journal is indexed on American Chemical Society's Chemical Abstracts Service (CAS)
The manuscript management system is completely online and includes a very quick and fair peer-review system, which is all easy to use. Visit http://www.dovepress.com/testimonials.php to read real quotes from published authors. 